\title{
Características dos Idosos em Área de \\ Abrangência do Programa Saúde da Família na Região Noroeste do Paraná: contribuições para a gestão do cuidado em enfermagem
}

Characteristics of Elderly People in an Area Assisted by the Family Health Program in the Northwest of Paraná:

contributions to nursing care management

\author{
Viviani Camboin Meireles \\ Enfermeira, Mestre em Enfermagem pela Universidade Estadual \\ de Maringá. \\ E-mail: vcmeireles®ibest.com.br \\ Laura Misue Matsuda \\ Enfermeira, Doutora em Enfermagem. Docente da Universidade \\ Estadual de Maringá. \\ Jorseli Ângela Henriques Coimbra \\ Enfermeira, Doutora em Saúde Pública. Docente da Universida- \\ de Estadual de Maringá. \\ Thais Aidar de Freitas Mathias \\ Enfermeira, Doutora em Saúde Pública. Docente da Universida- \\ de Estadual de Maringá.
}

\section{Resumo}

Este artigo é produto de um estudo do tipo descritivoexploratório e teve como principal objetivo investigar a população idosa atendida por uma Equipe de Saúde da Família (ESF) de uma área de Maringá-PR. Como resultado, constatou-se predominância de mulheres idosas na faixa etária de 60 a 79 anos; na faixa de 80 anos ou mais houve predomínio de homens. Dentre as mulheres, $81,8 \%$ possuem algum tipo de renda. Dentre os homens, esse número foi de $91,7 \%$. As doenças de maior prevalência entre os idosos de ambos os sexos foram à hipertensão arterial e a diabetes, seguida da Hipertensão Arterial e Diabete Mellitus como doenças associadas que corresponderam a 4 (12,1\%) das mulheres. Observou-se grande número de registros em branco em relação a este dado, sendo $25 \%$ entre os homens e 12,1\% entre as mulheres. Em relação à moradia, $82,5 \%$ moram com duas ou mais pessoas. $\mathrm{O}$ analfabetismo foi prevalente entre os homens. $\mathrm{O}$ conhecimento dos dados referentes às características dos idosos permite ao serviço de saúde gerenciar a assistência à saúde de maneira mais coerente e adequada à realidade vivida pela clientela. Considera-se que a estruturação e a execução das políticas de saúde ao idoso, fundamentadas no diagnóstico de problemas específicos, possibilitam ações e resultados efetivos. Palavras-chave: Idoso; Enfermagem; Cuidado; Gestão. 


\section{Abstract}

This descriptive-exploratory research aimed to investigate the elderly population assisted by one Family Health Team of an area of Maringá, a town located in the northwest of the state of Paraná. The results showed predominance of elderly women between 60 and 79 years old, but in the age group of 80 years of age or more, men predominated. Among the women, $81.8 \%$ have some kind of income; among the men, this number increases to $91.7 \%$. The most prevalent diseases among the patients of both sexes were arterial hypertension and diabetes, followed by arterial hypertension and diabetes mellitus as "doenças coadjuvantes", corresponding to 4 (12.1\%) women. We observed that a large number of records were not filled up in relation to this information: $25 \%$ among men and $12.1 \%$ among women. Regarding residence, $82.5 \%$ of the elderly live with two people or more. Illiteracy was predominant among the men. Knowledge of data about the elderly's characteristics allows the health service to manage the health assistence in a more coherent way, in tune with the clients' reality. We consider that the structure and execution of health policies focusing on the elderly, founded on the diagnosis of specific problems, enable effective actions and results.

Keywords: Elderly; Nursing; Care; Management.

\section{Introdução}

Envelhecer pode ser definido como um processo conseqüente de alterações no organismo, manifestado de forma variável e individual. Pode se referir a um fenômeno fisiológico, de comportamento social, ou ainda cronológico, isto é, a velhice surge com a progressão do tempo, da idade adulta até o fim da vida.

O homem, em desenvolvimento durante o ciclo da vida, é um ser biopsicossocial, passível de ser influenciado pelo ambiente físico, político e cultural em que vive, o qual pode facilitar ou dificultar seu processo de adaptação, acelerando ou retardando o envelhecimento (Rodrigues e col., 1996).

No Brasil, nas últimas décadas, iniciou-se um processo de inversão na característica populacional, tendo havido um decréscimo nas taxas de natalidade e mortalidade, o que ocasionou o aumento da população da faixa etária de 60 anos ou mais. Esse processo vem ocorrendo devido à diminuição relativa do contingente populacional nas faixas etárias mais jovens de zero a 14 anos, à ampliação da população na faixa etária de 15 a 59 anos e ao acréscimo na faixa de 60 anos ou mais.

Até meados do século passado a probabilidade de os brasileiros morrerem no auge da vida produtiva era um fato bastante concreto. Na atualidade, a expectativa de vida vem aumentando em virtude dos avanços científicos e tecnológicos, das melhores condições de higiene e saneamento básico, que reduziram, por exemplo, as mortes por doenças infecto-contagiosas.

Atualmente, envelhecer deixou de ser característica de países desenvolvidos, pois cerca da metade dos idosos do mundo vive em países em desenvolvimento. Segundo Schoueri e col. (200o), em 1980 a população idosa no Brasil correspondia a cerca de 7 milhões de pessoas com mais de 6 o anos. Em 1991, esse número passou para 11milhões, e as projeções para o ano de 2025 são de aproximadamente 34 milhões de indivíduos com mais de 60 anos, o que colocará o Brasil na sexta posição entre os países com maior número de pessoas idosas.

Fatos como o aumento da população idosa, influenciam o modo de gerir a atenção à saúde, visto que uma sociedade em processo de mudanças tem necessidade de adequação dos valores culturais, das políticas sociais e de saúde, de maneira a atender às neces- 
sidades e aos problemas decorrentes do envelhecimento populacional (Stevenson e col., 1997).

Para a Organização das Nações Unidas (ONU, 1982), o ser idoso difere entre países desenvolvidos e países em desenvolvimento. Nos primeiros, são consideradas idosas as pessoas com 65 anos ou mais, enquanto nos países em desenvolvimento, como é o caso do Brasil, são idosos aqueles com 60 anos ou mais. Essa definição foi estabelecida pela ONU, em 1982, por meio da Resolução 39/125, durante a Primeira Assembléia Mundial das Nações Unidas sobre o Envelhecimento da População.

Para a definição de população idosa, podemos utilizar a idade funcional, que avalia a idade de acordo com o desempenho funcional, ou a idade cronológica, que consiste nos anos vividos desde o nascimento. Segundo Roach (2003), o método cronológico é o mais utilizado, por ser o mais fácil; no entanto, não é o mais eficaz. Como exemplo, pode se comparar duas pessoas com a mesma idade cronológica e observar muitas discrepâncias funcionais, físicas, mentais e de saúde entre elas. Envelhecer pode ser definido como um processo conseqüente de alterações no organismo, demonstradas de forma variável e individual. Pode se referir a um fenômeno fisiológico, de comportamento social, ou ainda cronológico, isto é, que surge com a progressão do tempo da idade adulta até o fim da vida.

O processo de envelhecimento provoca no organismo modificações biológicas (morfológicas) - como o aparecimento de rugas e cabelos brancos -; fisiológicas, que acarretam alterações das funções orgânicas; e bioquímicas, que aparecem por meio das transformações das reações químicas que se processam no organismo (Rodrigues e col., 1996).

As modificações psicológicas ocorrem principalmente quando, ao envelhecer, a pessoa precisa adaptar-se a cada situação nova do seu cotidiano. Dentre essas mudanças, estão as transformações sociais, por conseguinte, a diminuição da produtividade e, principalmente, do poder físico e econômico (Silva, 2005).

De acordo com Vermelho e Monteiro (2002), a transição demográfica está intimamente ligada à transição epidemiológica, ou seja, devido ao aumento da população idosa, ocorre maior índice de morbimortalidade por doenças crônico-degenerativas e suas seqüelas. Mediante a esse fato, fazem-se necessárias novas formas de construção do conhecimento e sua relação com o mun- do do trabalho, de modo a ajustar os profissionais e os serviços de saúde para uma efetiva implementação de políticas públicas adequadas aos idosos.

Segundo Lima-Costa e col. (2003), uma das conseqüências do crescimento da população idosa é o aumento da demanda por serviços de saúde e sociais. Dessa forma, as internações nessa faixa etária, bem como o tempo de ocupação do leito, são mais elevadas. Assim, a inadequação das políticas sociais ganha visibilidade, pois a possibilidade de envelhecimento está extremamente relacionada ao acesso a condições dignas de vida e renda, fator determinante da sua qualidade de vida ao envelhecer.

Estudos epidemiológicos têm demonstrado que doenças e limitações não são conseqüências inevitáveis do envelhecimento, mas dependerão do acesso que o indivíduo tem aos serviços preventivos, que orientam para a redução de fatores de risco e levam à adoção de hábitos de vida saudáveis. Dependerão igualmente, da visão de mundo da sociedade em que está inserido, bem como das condições socioeconômicas do indivíduo (Bretas, 2003).

No Brasil, na tentativa de melhorar as condições de atendimento, a partir dos anos 1990 foi criado pelo Ministério da Saúde, o Programa Saúde da Família (PSF), visando à reversão do modelo assistencial até então vigente, por meio da mudança do foco de atenção baseado no modelo biomédico, hospitalocêntrico. Conforme o novo modelo, a família passou a ter um papel fundamental nesse processo e o domicílio passou a ser um cenário de assistência, promoção à saúde e prevenção de doenças (Souza, 200o).

De acordo com Souza (200o), as unidades da Equipe de Saúde da Família (ESF), por meio de suas equipes multiprofissionais, compostas no mínimo de médico, enfermeiro, auxiliar de enfermagem e agentes comunitários de saúde (ACS), devem constituir o primeiro contato da população com os serviços de saúde e assim estabelecer uma relação de referência e contra-referência com outros níveis de assistência.

No âmbito da enfermagem, o processo de trabalho do enfermeiro tem implícitas as dimensões cuidar e administrar/gerenciar (Trevizan, 1988). Nesse sentido, o conhecimento das características locais da clientela, para uma atuação efetiva de toda a equipe de saúde, faz-se importante.

Mathias e col. (2004), ao realizarem um estudo em 
relação à mortalidade por doenças cardiovasculares, consideraram que o conhecimento da situação de saúde da população é importante, pois pode propiciar a geração de hipóteses causais além de contribuir para a elaboração de programas e políticas de saúde.

Considerando as diretrizes do PSF, as equipes devem realizar o cadastramento das famílias de sua área de abrangência, que contempla o atendimento de 800 a 1000 famílias por ESF. Cada área é dividida em microáreas, as quais possuem um ACS, responsável pelo cadastramento das famílias mediante visitas domiciliares. Este cadastramento tem por finalidade realizar um diagnóstico de saúde da comunidade, auxiliando no planejamento e na organização das ações de promoção e prevenção da saúde (Souza, 200o).

Segundo Kalache (1993), devem ser realizadas pesquisas que subsidiem a tomada de decisão e dirijam as ações e prioridades para políticas públicas relativas à terceira idade. Dentre essas recomendações, pode-se destacar a necessidade de se realizarem análises multidimensionais visando ao estabelecimento de um diagnóstico e de indicadores básicos acerca da população idosa, tais como: a) idade, sexo, nível educacional e condições socioeconômicas; b) descrição dos problemas e necessidades que afetam a população-alvo, com ênfase naqueles que influenciam o estado de saúde e o bem-estar geral; c) a busca de dados de morbidade/incapacidade.

No contexto atual em que emerge o Programa Saúde da Família, considerando que os arranjos familiares são importantes apoios no processo saúde-doença, e mediante a necessidade de se conhecer a real situação de saúde da população idosa brasileira, é que nos motivamos a buscar dados relacionados a algumas características dos idosos residentes na área de abrangência de uma Unidade Básica de Saúde (UBS) de um município da região Noroeste do Paraná. Outro fator é que os resultados levantados poderão subsidiar ações de melhorias na gestão do cuidado a essa clientela.

O objetivo geral deste estudo consiste em investigar a população idosa atendida por uma Equipe de Saúde da Família (ESF) de uma área de Maringá-PR. Como objetivos específicos, temos: descrever algumas características sócio-demográficas e epidemiológicas da população idosa assistida por uma ESF; discutir acerca da gestão do cuidado de enfermagem a essa população e sugerir ações de melhorias para o cuidado ao idoso.

\section{Material e Método}

Este trata-se de um estudo descritivo-exploratório, de caráter epidemiológico. Segundo Lima-Costa e Barreto (2003), os estudos descritivos têm como objetivo determinar a distribuição de doenças ou condições relacionadas à saúde, segundo o tempo, o lugar ou as características dos indivíduos. Podem fazer uso de dados secundários, examinando a incidência ou a prevalência de uma doença ou condição relacionada à saúde, a qual varia de acordo com determinadas características, como: sexo, idade, escolaridade, renda, dentre outras.

Este trabalho teve como critério para a definição de população idosa a idade cronológica. Optou-se por estudar a faixa etária de 60 anos ou mais de idade, considerada etapa inicial do envelhecimento, segundo a Organização das Nações Unidas (ONU, 1982) para países em desenvolvimento, como o Brasil.

A coleta dos dados foi realizada no período de abril a junho de 2005 em uma cidade de médio porte, localizada na região Noroeste do Estado do Paraná.

No município em estudo, a rede pública de saúde é formada por 23 Unidades Básicas de Saúde (UBS) um Hospital Universitário e um Hospital Municipal. Atualmente, as UBS contam com 64 equipes do Programa de Saúde da Família.

Este estudo se insere em um Projeto de Extensão institucional da Universidade Estadual de Maringá e foi desenvolvido no bairro da área de abrangência do PSF. Levando em consideração a necessidade de um trabalho voltado para a promoção da saúde da população, o referido projeto é constituído por uma equipe multidisciplinar (Enfermagem, Educação Física, Pedagogia, Psicologia, Farmácia) e é desenvolvido com o objetivo de promover, manter e recuperar a saúde das pessoas da comunidade do bairro.

A estratégia utilizada para a coleta de dados foi a consulta a documentos. Os dados foram obtidos a partir de fontes secundárias como da Ficha A, que é a Ficha de Cadastro de Famílias, preenchida pelos ACSs.

$\mathrm{Na}$ área em estudo existiam 189 famílias cadastradas com 656 pessoas. Destas, 57 encontravam-se com 60 anos ou mais de idade.

O procedimento para a coleta de dados ocorreu da seguinte forma:

- Por comparação dos dados obtidos por meio da confe- 
rência dos dados da Ficha A, com o Caderno de Anotações dos ACSs e de visitas domiciliares.

- Por meio de consulta das informações que compõem o tema em estudo.

- Com a identificação, a partir dos registros efetuados nas fichas de cadastros das famílias, de todas as pessoas com 60 anos ou mais de idade.

- Pela compilação dos dados, em planilhas construídas especificamente para este estudo, referentes a idade, sexo, alfabetização, morbidade e ocupação.

Para a apresentação e a discussão dos resultados, os dados foram agrupados e contabilizados por freqüência e porcentagens simples. A apresentação dos dados será efetuada na forma de tabelas e figuras.

\section{Resultados e Discussão}

\section{Características Demográficas}

Considerando a continuidade das tendências para as taxas de fecundidade e longevidade, as estimativas para os próximos 20 anos indicam que o número de idosos no Brasil poderá exceder 30 milhões, representando quase $13 \%$ da população (Veras, 2004).

Na Tabela 1 consta a distribuição dos idosos de acordo com o sexo e a faixa etária.

\section{Tabela I - Distribuição dos idosos, segundo a faixa etária e sexo. PR, 2005}

\begin{tabular}{|l|c|c|c|c|c|c|c|c|}
\hline Idade & \multicolumn{2}{|c|}{60 a 69 anos } & \multicolumn{2}{c|}{70 a 79 anos } & \multicolumn{3}{c|}{80 anos ou mais } & \multicolumn{3}{|c|}{ Total } \\
\cline { 2 - 10 } Sexo & N & $\%$ & N & $\%$ & N & $\%$ & N & $\%$ \\
\hline Feminino & 22 & 56,4 & 9 & 69,2 & 2 & 40,0 & 33 & 57,9 \\
\hline Masculino & 17 & 43,6 & 4 & 30,8 & 3 & 60,0 & 24 & 42,1 \\
\hline Total & 39 & 100,0 & 13 & 100,0 & 5 & 100,0 & 57 & 100,0 \\
\hline
\end{tabular}

Observa-se na Tabela 1 que há maior percentual de mulheres em relação a homens nas faixas etárias de 6 o a 69 anos $(56,4 \%)$ e 70 a 79 anos (69,2\%). Na faixa etária de 80 anos ou mais, a situação inverte-se, revelando o predomínio de homens (6o\%). Vale lembrar que esse resultado pode ter sido influenciado pelo número pequeno de pessoas nessa faixa de idade na população estudada.
Segundo Veras (2004), a maioria dos idosos brasileiros é do gênero feminino. Em 1991, eram 5,7 milhões de mulheres idosas, passando para 8 milhões em 200o, estimando-se um total de 8,5 milhões em 2002; os homens idosos representavam 4,9 milhões em 1991, 6,5 milhões em 2000, e 6,9 milhões em 2002.

De acordo com Veras (2004), apesar de a expectativa de vida ao nascimento contemplar ambos os sexos, isso não ocorre de maneira uniforme. A população brasileira, entre os anos de 1920 a 1982, experimentou um aumento de quase 30 anos de vida, entretanto esse aumento foi mais significativo para as mulheres do que para os homens. Esses dados são baseados em fatores, como: maior exposição do sexo masculino a acidentes de trabalho e morte por causas externas, diversidade de consumo de tabaco e álcool - que estão associados às mortes externas -, neoplasmas e doenças cardiovasculares; condutas em relação às doenças.

As mulheres em geral são mais atentas aos sintomas, possuem um conhecimento maior das doenças, expressam melhor os seus sintomas e procuram mais os serviços de saúde, além disso, a mortalidade por causas obstétricas diminuiu significativamente em relação ao passado (Veras, 2004). Vários desses fatores contribuem para a feminilização da velhice, corroborando com o achado da Tabela 1, no qual o percentual de mulheres idosas é maior que o de homens.

Ainda segundo Veras (2004), vários fatores de risco com predomínio no sexo masculino se estenderão em breve às mulheres, devido à incorporação de alguns valores na luta por igualdade de oportunidades e ampliação de espaço.

Ao nosso ver, essa predominância tem repercussões importantes nas demandas por políticas públicas e na gestão do cuidado, pois se acredita que a maioria dessas mulheres atualmente, seja viúva, com menor escolaridade e renda, com grande possibilidade de experimentar piores condições de saúde, requerendo maior assistência tanto do Estado como das famílias.

\section{Características socioeconômicas}

Segundo Lima-Costa e Barreto (2003), a situação socioeconômica desempenha papel essencial na determinação da saúde de indivíduos e populações. Estudos realizados por esses autores mostram que os idosos com baixa renda apresentam piores condições de 
saúde (interrupção de atividades devido a problemas de saúde, doenças crônicas, pior função física) e menor procura por serviços de saúde (médicos e odontológicos).

Uma das conseqüências da aposentadoria é a diminuição do status social do idoso que, na maioria das vezes, arca com os custos de manutenção de sua casa. Segundo Assis (2004), o problema da aposentadoria no Brasil é que se subtrai valor de um salário que já era baixo, ocasionando uma velhice em situação financeira pior do que quando se trabalhava, impondo ao idoso a redefinição de seu padrão de vida, a busca de nova forma de renda complementar ou a forçosa adaptação ao declínio da situação econômica.

Além disso, as oportunidades de trabalho remunerado para complementar a renda do idoso são mínimas, devido, muitas vezes, ao baixo nível de formação educacional dos idosos e da competição com os jovens em um mercado de trabalho cada vez mais competitivo.

Na tabela 2, a seguir, consta a distribuição de freqüência dos idosos segundo o sexo e a ocupação.

\section{Tabela 2 - Distribuição dos idosos estudados, segundo sexo e ocupação. Maringá, PR, 2005}

\begin{tabular}{|l|c|c|c|c|c|c|}
\multirow{2}{*}{ Ocupação } & \multicolumn{5}{|c|}{ Sexo } & \multicolumn{2}{c}{ Total } \\
\cline { 2 - 7 } & \multicolumn{2}{|c|}{ Feminino } & \multicolumn{2}{|c|}{ Masculino } & \multicolumn{2}{c|}{ N } \\
\cline { 2 - 7 } & N & $\%$ & N & $\%$ & N & $\%$ \\
\hline Do lar & 6 & 18,2 & - & 0,0 & 6 & 10,5 \\
\hline Pensionista & 5 & 15,2 & - & 0,0 & 5 & 8,8 \\
\hline Aposentado(a) & 19 & 57,6 & 22 & 91,7 & 41 & 71,9 \\
\hline Trabalho ativo & 3 & 9,1 & 2 & 8,3 & 5 & 8,8 \\
\hline Total & 33 & 100,0 & 24 & 100,0 & 57 & 100,0 \\
\hline
\end{tabular}

Observa-se que, dentre as 33 mulheres, 27 (81,8\%) possuem algum tipo de renda, sendo $5(15,2 \%)$ relacionados à pensão, e 19 (57,6\%) à aposentadoria e $3(9,1 \%)$ recebem renda devido ao seu trabalho. Seis $(18,2 \%)$ idosas não são aposentadas e realizam tarefas do lar. Das $3(9,1 \%)$ que não são aposentadas e ainda trabalham, 1 (3,03\%) é telefonista, 1 (3,03\%) é professora e 1 (3,03\%) é diarista.

Sabe-se que as mulheres, apesar das mudanças no mercado de trabalho e da educação formal, devido a fatores culturais e sociais, ainda têm acesso restrito nessas áreas. Desse modo, a sua inserção no papel doméstico é ainda elevada como se vê nos resultados deste estudo.

Entre o sexo masculino, observa-se que $22(91,7 \%)$ são aposentados, 2 (8,3\%) embora sejam aposentados ainda trabalham e $2(8,3)$ não são aposentados. A situação previdenciária mostra que entre 57 idosos de ambos os sexos, 41 (71,9\%) conseguiram se aposentar. Esse percentual, somado ao dos que recebem pensão, aumenta para 46 (8o,7\%). Onze (19,3\%) não recebem benefício previdenciário sendo que, destes, 6 (10,5\%) realizam atividades do lar e $5(8,8 \%)$ ainda trabalham.

Em relação à moradia, $47(82,5 \%)$ dos idosos estudados moram com duas ou mais pessoas na mesma casa. Conforme dados do Instituto Brasileiro de Geografia e Estatística - IBGE (Brasil, 2003), o número médio de pessoas por família consiste em 3,4 indivíduos. Na população idosa, segundo essa mesma fonte, o número de domicílios unipessoais, ou seja, domicílio onde mora apenas uma pessoa, corresponde a $15,4 \%$ do total. Detectou-se também que dentre os idosos, $10(17,5 \%)$ moram sozinhos, sendo a maioria mulheres (90\%).

A predominância de mulheres que residem sozinhas é mencionada no estudo de Lebrão e Laurenti (2005) ao realizarem estudos do SABE (Saúde, bemestar e envelhecimento). Ao coletar informações sobre as condições de vida dos idosos (com 60 anos ou mais), residentes na área urbana do Município de São Paulo, os autores detectaram que em relação ao fator gênero, 25,3\% das mulheres viviam sozinhas e apenas $13,8 \%$ dos homens se encontravam em igual situação.

Ainda segundo o estudo de Lebrão e Laurenti (2005), a maioria dos idosos (86,8\%) vivia acompanhada, porém esse valor caia à medida que eles envelheciam, pois entre aqueles com 80 anos ou mais, $21,5 \%$ moravam sozinhos.

Morar sozinho não constitui um problema em si, já que pode ser uma opção - somente possível, em princípio, se as condições financeiras e de saúde o permitirem. Entretanto, é considerada uma situação de risco, caso o idoso possua algum comprometimento funcional, tendo-se em vista a possibilidade de perda da autonomia e inadequado suporte familiar. As dificuldades se relacionam ao sentimento de solidão, à má-alimentação (qualitativa e quantitativa), aos cuidados com sua 
saúde, a higiene pessoal e domiciliar (Anderson, 1998).

Os idosos que vivem sozinhos merecem atenção específica dos membros da equipe de saúde para verificar a existência de incapacidades que impeçam a sua sobrevivência sem apoio de outras pessoas. Nesse sentido, os profissionais da saúde e, em particular, a enfermagem deverá gerir o cuidado do idoso no sentido de prever e prover, sempre que possível, os meios e os recursos existentes na comunidade, para a continuidade do cuidado.

Tem-se então que, no contexto da gestão do cuidado ao idoso, as atividades dos profissionais de saúde e em especial a da enfermagem, devem ser desenvolvidas com o intuito de contribuir para a prevenção de doenças e complicações. Assim, ao idoso que mora sozinho, deve-se, sempre que possível, promover o convívio com os familiares, com a comunidade, preservando a sua autonomia e buscando a sua máxima independência funcional.

A perda do apoio familiar ao idoso acontece geralmente devido às mudanças na composição familiar, nas modificações socioculturais e econômicas. Antes, famílias numerosas conviviam no mesmo ambiente domiciliar e a presença do idoso representava um status social elevado. Com o transcorrer dos anos a família numerosa foi substituída por uma família nuclear com pai, mãe e poucos filhos. Detecta-se ainda que as mulheres, que geralmente assumiam o papel de cuidadoras, estão no mercado de trabalho e já não têm mais "tempo" para cuidar de seus idosos.

Mediante a essa realidade, os profissionais de saúde necessitam prover ações de promoção e prevenção que devem se iniciar no domicílio por meio da higiene, ventilação, alimentação, e por meio das imunizações e detecção precoce das doenças, pois quando a pessoa está doente ou acamada é possível prevenir a piora do quadro, evitando-se imobilizações e perda de funcionalidade.

A situação do idoso na sociedade brasileira necessita de maior ênfase no que se refere à qualidade de vida e ao acesso aos serviços básicos que atendam as suas necessidades individuais e sociais. Assim, ao pensar no gerenciamento do cuidado ao idoso, o enfermeiro e a equipe devem realizar programas de orientação, informação, suporte informal e familiar e apoio de profissionais capacitados para atender as especificidades do cuidado ao idoso.
Muitos idosos encontram-se em dificuldade de manter sua autonomia e sua independência, devido a precárias condições de vida e de saúde, em face das incapacidades físicas, muitas vezes passíveis de prevenção e reabilitação. Outro fator agravante é o desequilíbrio psico-emocional gerado por ausência de relações sociais e afetivas.

Quanto à escolaridade, conforme dados do Instituto Brasileiro de Geografia e Estatística - IBGE (Brasil, 2003), os anos de estudo dos brasileiros se situam, na maioria, entre quatro e sete anos. Entre os idosos, essa média, é de 2,7 anos.

Acreditamos que a escolaridade dos idosos influencia o seu poder aquisitivo e, por conseguinte, as oportunidades e o acesso aos serviços que proverão suas necessidades biopsicossociais, dentre as quais se encontram a alimentação, habitação, saúde e lazer.

A seguir, na Figura 1, consta a distribuição dos idosos de acordo com o sexo e alfabetização.

\section{Figura I - Distribuição dos idosos estudados segundo o sexo e alfabetização. Maringá, PR, 2005}

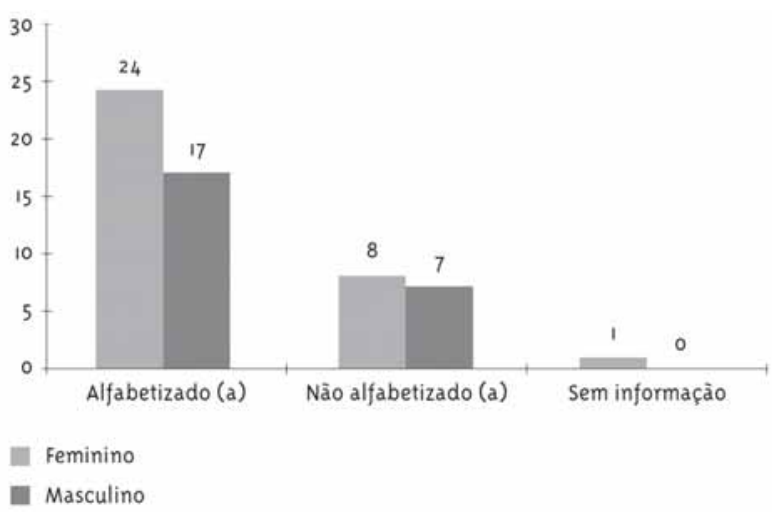

De acordo com a Figura 1, observa-se que no grupo em estudo, 8 ( $24,2 \%)$ das mulheres não eram alfabetizadas e 7 (29,2\%) dos homens se encontravam nessa condição.

Neste estudo, consideramos alfabetizados os indivíduos que sabiam escrever e como analfabetos aqueles que não sabiam ler e escrever.

O baixo nível de escolaridade deve ser considerado no planejamento de programas e ações, tanto no nível coletivo quanto no individual. O nível educacional interfere diretamente no desenvolvimento da consciência sanitária, na capacidade de entendimento do 
tratamento prescrito e na prática do autocuidado, interferindo diretamente na relação médico-paciente.

Segundo os dados do IBGE (Brasil, 200o), da década de 1930 até os anos de 1950, o ensino fundamental era restrito a segmentos sociais específicos. Até os anos 1980, os baixos níveis de escolaridade não eram considerados como fator limitador do desenvolvimento, sendo aceitável pelo modelo econômico, industrial e tecnológico do país. Acredita-se que isso pode ser explicado devido aos valores culturais e sociais da primeira metade do século passado, em que as mulheres assumiam o papel domiciliar, aliado a dificuldade de acesso ao sistema educacional, pois possivelmente essa geração de idosos, dessa região, vivia na zona rural quando estava em idade de escolarização. Conseqüência disso é o baixo nível da escolaridade da população idosa.

Sabe-se que os baixos níveis de escolaridade associados a fatores socioeconômicos e culturais contribuem para o aparecimento de doença, pois esses fatores podem dificultar a obtenção de informações e a conscientização das pessoas sobre a relevância dos cuidados com a saúde ao longo da vida, a necessidade da adesão ao tratamento e a manutenção de hábitos saudáveis.

Na figura 2, a seguir, consta a distribuição por sexo, das doenças que acometem os idosos, participantes deste estudo.

\section{Figura 2 - Doenças dos idosos estudados segundo o sexo. Maringá, PR, 2005}

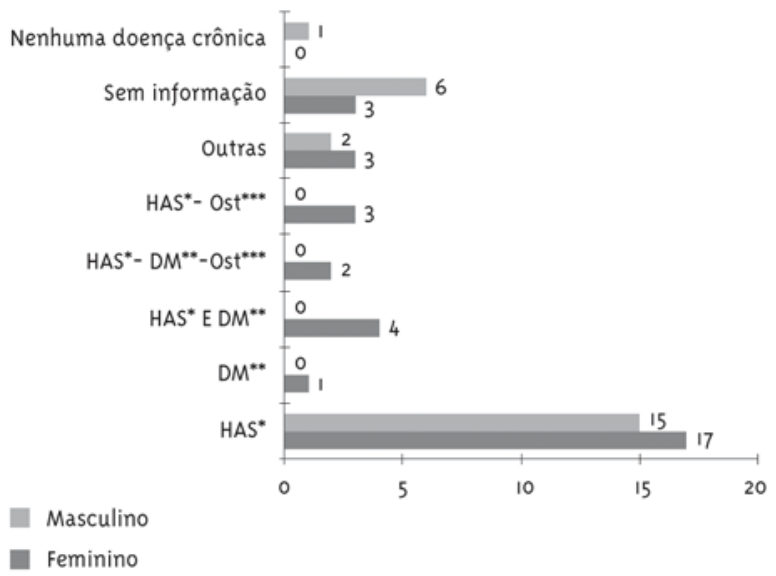

Nota: *HAS: Hipertensão Arterial Sistêmica.

**DM: Diabetes Mellitus.

${ }^{* * *}$ Ost: Osteoporose.
Esses dados mostram que a hipertensão arterial é a doença que mais acomete os idosos em estudo. Dentre as 33 mulheres, 17 (51,5\%) apresentam hipertensão arterial, 4 (12,1\%) apresentam hipertensão arterial e diabetes mellitus, como doenças associadas, 2 $(6,1 \%)$ apresentam hipertensão arterial, diabetes mellitus e osteoporose, 3 (9,1\%) apresentam hipertensão arterial e osteoporose, 1 (3,0\%) apresenta diabetes mellitus. Doenças não mencionadas: 1 (3,0\%) com etilista, 1 (3,0\%) com epilepsia e 1 (3,0\%) com dislepidemia e osteoporose, sendo que 3 (9,1\%) das mulheres não possuíam registros relativos a esse dado.

Vários motivos dificultaram a coleta de informações deste estudo. Dentre eles constam a pouca disponibilidade e o mau funcionamento de computadores, a falta de organização da ESF e a desatualização do banco de dados do SIAB (Sistema de Informação de Atenção Básica). Observou-se que os profissionais realizavam suas anotações relacionadas às patologias na ficha A e as alterações no quadro de saúde eram marcadas no Caderno de anotações do ACS. Isso tudo inviabiliza o armazenamento de dados e o sistema de informação, essencial ao (re)planejamento e a gestão dos serviços de saúde fica comprometido.

Ainda em referência ao sistema de informações, é necessário que todos os profissionais da Equipe da Saúde da Família conheçam e utilizem os dados do SIAB a fim de traçar estratégias, definir metas e identificar intervenções necessárias para a atenção da população das suas respectivas áreas de cobertura. A avaliação do resultado do trabalho desenvolvido pela equipe também consiste em fator de suma importância.

No sexo masculino, houve também a prevalência de hipertensão arterial em 15 idosos (62,5\%). Entre as outras doenças que acometem os idosos deste estudo estão o câncer 1 (4,2\%) e o acidente vascular cerebral também 1 (4,2\%). Seis fichas (25\%) não possuíam registros das informações relacionadas a doenças e 1 informava não possuir doença crônica.

A pressão arterial é exercida pela pressão do sangue nas artérias e os valores normais para os adultos variam de 100/6o mmHg a 140/90 mmHg, sendo considerado hipertensão arterial valores acima deste limite (Smeltzer e Bare, 1998).

Em relação à hipertensão arterial sistêmica, os dados levantados neste estudo estão um pouco acima daqueles encontrados em estudos nacionais (prevalên- 
cia de 51,5\% entre as mulheres e 62,5\% dos homens), sendo que esta é uma doença de alta prevalência em nosso país, atingindo cerca de $20 \%$ da população adulta jovem e 50\% da população idosa. A hipertensão arterial sistêmica possui forte relação com $80 \%$ dos casos de acidente vascular encefálico (AVE) e 6o\% dos casos de doença isquêmica do coração (Brasil, 2001). Constitui, sem dúvida, o principal fator de risco para as doenças cardiovasculares, cuja principal causa de morte, o AVE, tem como origem a hipertensão não controlada.

O diabetes mellitus é um grupo de distúrbios heterogêneos caracterizado por níveis elevados de glicose no sangue ou hiperglicemia. Pode ser do tipo I, ou insulino-dependente, que acomete cerca de $5 \%$ a $10 \%$ das pessoas com diabetes e caracteriza-se por início súbito, geralmente antes dos 30 anos de idade. O diabetes mellitus tipo II, ou insulino-independente, acomete de $90 \%$ a $95 \%$ das pessoas com diabetes e ocorre mais freqüentemente em pessoas com mais de 30 anos de idade (Smeltzer e Bare, 1998).

No grupo em estudo, 12,1\% das mulheres apresentam hipertensão arterial e diabetes. A hipertensão arterial e o diabetes mellitus constituem os principais fatores de risco populacional para as doenças cardiovasculares' e são agravos de saúde pública dos quais até $80 \%$ dos casos podem ser tratados na atenção básica (Brasil, 2001).

Segundo dados do Ministério da Saúde, a população de portadores de diabetes mellitus estimada no Brasil é de cinco milhões. A doença atinge todas as faixas etárias, independente de raça, sexo ou condições socioeconômicas, e já representa a quarta principal causa básica de morte. Na população adulta, sua prevalência é de 7,6\% (Brasil, 2001).

A osteoporose esteve presente em 12,1\% das mulheres que participaram deste estudo. De acordo com Carvalho e col., (2004), a osteoporose é uma doença sistêmica que resulta em reduzida massa óssea e aumento da porosidade do osso, levando à fragilidade mecânica e conseqüente predisposição a fraturas. Segundo esses autores, a osteoporose atinge ambos os sexos, porém a maior incidência está entre as mulheres após a menopausa, em função do declínio acentuado dos hormônios. Os autores também apontam que a etiologia da osteoporose é multifatorial, visto que inclui fatores relacionados à hereditariedade, etnia, idade avançada, sexo feminino, baixo peso corporal, defi- ciência hormonal, excessivo consumo de álcool, inatividade física, tabagismo e fatores nutricionais.

Em relação às doenças crônicas em famílias residentes na região Norte de Maringá, estudos realizados por Marcon e col. (2004) também identificaram como doenças mais freqüentes a hipertensão arterial, o tabagismo, o diabetes e o alcoolismo. Esses autores referem que as doenças crônicas apresentam peculiaridades, como duração e risco de complicações, o que exige um rigoroso esquema de cuidados. Afirmam também que a prevenção de seqüelas constitui em um dos pontos fundamentais da atenção ao doente crônico, visto que elas podem resultar em incapacidades funcionais permanentes, principalmente quando o paciente é idoso.

Com o envelhecimento populacional, tem-se um aumento da prevalência de doenças crônicas e incapacitantes e uma mudança de paradigma na saúde pública. Devido à relevância desse problema, a Organização Mundial da Saúde criou o Relatório Mundial para Cuidados Inovadores para Condições Crônicas (OMS, 2003). Segundo esse Relatório o tratamento não se baseia na etiologia da doença, mas nas exigências que ela impõe ao sistema. Assenta ainda que para o alcance de resultados satisfatórios, os pacientes, o grupo de apoio da comunidade e a equipe de atenção à saúde devem ser mantidos informados, motivados e capacitados a trabalharem em parceria, ou seja, a atenção deve ser integrada envolvendo o paciente, a comunidade e a organização de saúde.

O Estatuto do Idoso (Brasil, 2003), aponta que a mudança no perfil epidemiológico do idoso acarreta grandes despesas, pois o idoso exige mais serviços de saúde, com internações hospitalares freqüentes e o tempo de ocupação do leito, normalmente, é maior do que em outras faixas etárias. Em geral, as doenças dos idosos são crônicas e múltiplas, perduram por vários anos e exigem permanente acompanhamento das equipes multidisciplinares e intervenções contínuas. Como agravante, tem-se ainda a falta de integração e comunicação entre os serviços dos diversos níveis de assistência, falhas no gerenciamento do cuidado e inadequação das políticas sociais, que são fatores determinantes da qualidade do cuidado.

O envelhecimento não deve ser encarado como números e repercussões para a previdência social, pois a sociedade como um todo tem responsabilidade 
no que se refere ao cuidado com o idoso. Observa-se, no entanto, que muitos serviços oferecidos a essa clientela, embora com objetivos de manter sua autonomia e independência, terminam por obter o oposto do que se propõe, aumentando a dependência e tirando do idoso a confiança em seus potenciais. Fatos desse tipo podem ser observados quando não se dá orientações em relação ao seu autocuidado, por acreditar que ele não possui capacidade de aprender. Assim, o profissional toma decisões de acordo com as suas próprias convicções, supondo que é o melhor para o idoso, não permitindo que ele faça suas escolhas.

Os cuidados com as pessoas idosas devem visar à recuperação ou à manutenção do seu estado de saúde, com perspectiva de vida ativa, junto aos familiares e à comunidade, com a máxima independência e autonomia possível.

O gerenciamento do cuidado do idoso envolve o (re)planejamento, coordenação e monitoramento dos serviços, de forma que venham ao encontro das suas necessidades e expectativas, no sentido de prevenir ou amenizar os riscos referentes aos problemas físicos, emocionais e funcionais que podem dificultar ou impedir sua vida comunitária independente.

\section{Considerações Finais}

Este estudo teve como objetivos descrever características demográficas e epidemiológicas da população idosa e também discutir acerca da gestão do cuidado de enfermagem ao idoso.

De acordo com os resultados, existe predomínio de pessoas com idade média de 68,8 anos e maior proporção de mulheres em relação a homens nas faixas etárias de 60 a 69 anos (56,4\%). Na faixa etária de 80 anos ou mais, a situação é diferente, revelando o predomínio de homens (60\%) em relação a mulheres.

Dentre as 33 mulheres, que compuseram a amostra deste estudo, 27 ( $81,8 \%)$ possuem algum tipo de renda, sendo $5(15,2 \%)$ relacionados à pensão, e 19 (57,6\%) à aposentadoria. Entre os homens, 91,7\% são aposentados e 11 (19,3\%) dos idosos não recebem benefício previdenciário.

Dos 57 idosos, 47 (82,5\%) moram em domicílios com duas ou mais pessoas, porém um percentual importante $(17,5 \%)$ mora sozinho. A doença de maior incidência foi a hipertensão arterial, acometendo 51,5\% das mulheres e $62,5 \%$ dos homens, seguida da hipertensão arterial e diabete mellitus como doenças coadjuvantes em $4(12,1 \%)$ mulheres. Vale destacar o grande número de fichas sem esse registro, o que correspondeu a $25 \%$ entre os homens e 9,1 entre as mulheres.

Considerar o idoso em suas múltiplas interfaces é uma tarefa difícil, porém de extrema urgência, para subsidiar a gestão do cuidado a essa clientela de maneira a preservar a sua independência e promover melhor qualidade de vida. Assim sendo, para a gestão do cuidado ao idoso, sugere-se que:

- a equipe de saúde e, em especial a da enfermagem, conheça cada idoso em sua unicidade e singularidade, visto que ações dessa natureza facilitam o planejamento dos cuidados no atendimento de suas necessidades e especificidades. Nesse âmbito, os problemas devem ser tratados e as habilidades sempre reforçadas.

- o enfermeiro, como gestor do serviço, promova a capacitação dos profissionais para prestar assistência de maneira competente e com qualidade, respeitando os idosos nas suas limitações, desfazendo mitos, preconceitos e estereótipos que envolvem o processo de envelhecimento e comprometem a qualidade da assistência prestada a essa clientela.

- o gerenciamento do cuidado do idoso envolva o (re)planejamento, a coordenação e o monitoramento dos serviços de forma que atendam as necessidades dos idosos que apresentam ou tem risco de apresentar problemas físicos, emocionais e funcionais, minimizando assim as dificuldades de se ter uma vida comunitária independente.

- a Unidade Básica de Saúde (UBS) seja o primeiro local de acesso aos serviços de saúde também para o idoso. Assim sendo, a UBS necessita fornecer serviços de qualidade, com base em um sistema de referência e contra-referência integrado e resolutivo.

- o enfermeiro que gerencia o cuidado avalie as necessidades e os recursos disponíveis, de forma a proporcionar ao idoso e à sua família todo atendimento possível. - o enfermeiro prime pelo preenchimento correto e atualizado das Fichas de Cadastros das famílias e dos idosos assistidos.

Com a criação do Programa de Saúde da Família pelo Ministério da Saúde (Brasil, 2001), ocorreu um 
processo de descentralização de recursos e desenvolvimento da gestão local, na qual os cargos gerenciais geralmente são exercidos por enfermeiros, que devem ter clareza sobre as mudanças ocorridas na sociedade, seu impacto sobre a organização para que se possam planejar as ações de modo efetivo.

Considera-se que os objetivos estabelecidos para este estudo foram alcançados visto que foi possível conhecer parte da realidade dos idosos de uma equipe do Programa Saúde da Família, com ênfase nas suas características sociais, econômicas e epidemiológicas. A discussão e a elaboração de sugestões acerca da gestão do cuidado de enfermagem ao idoso também foram possibilitadas.

\section{Referências}

ANDERSON, M. I. P. Saúde e condições de vida do idoso no Brasil. Textos sobre Envelhecimento, Rio de Janeiro, v. 1, n. 1, nov. 1998. Disponível em: <http:// www.unati.uerj.br/tse/index.php>. Acesso em: 5 jun. 2005 .

ASSIS, M. Aspectos sociais do envelhecimento. In: SALDANHA, A. L.; CALDAS, C. P. (Org.). Saúde do idoso: a arte de cuidar. 2. ed. Rio de Janeiro: Interciência, 2004. p. 11-21.

BRASIL. Lei ${ }^{0} 10.741$, de $1^{\circ}$ de outubro de 2003 . Dispõe sobre o estatuto do idoso e dá outras providências. Disponível em : <http://direitodoidoso. braslink.com/o5/estatuto_do_idoso.pdf>. Acesso em: 9 jul. 2005 .

BRASIL. Ministério da Saúde. Departamento de Atenção Básica. Guia prático do Programa de Saúde da Família. Brasília, DF, 2001.

BRETAS, A. C. Cuidadores de idosos e o Sistema Único de Saúde. Revista Brasileira de Enfermagem, Brasília, DF, v. 56, n. 3, p. 298-301, maio/jun. 2003.

CARVALHO, C. M. R. G.; FONSECA, C. C. C.; PEDROSA, J. I. Educação para a saúde em osteoporose com idosos de um programa universitário: repercussões. Cadernos de Saúde Pública, Rio de Janeiro, v. 2o, n. 3, p. 719-726, maio/ jun. 2004 .
INSTITUTO BRASILEIRO DE GEOGRAFIA E ESTATÍSTICA. Censo demográfico de 2003. Disponível em: <http://www.ibge.gov.br/>. Acesso em: 20 jun. 2005 .

\section{INSTITUTO BRASILEIRO DE GEOGRAFIA E} ESTATÍSTICA. Perfil dos idosos responsáveis pelos domicílios no Brasil - 200o. Estudos e Pesquisas: Informação Demográfica e Socioeconômica, Rio de Janeiro, n. 9, p.3-9, 2002.

KALACHE, A. Perspectives: what are the implications of population ageing in the foreseeable future. In: FUTURE health of aging population. Geneva: OMS, Unit of Monitoring Evaluation and Projection Methodology, 1993. p.20-27.

LEBRÃO, M. L.; LAURENTI, R. Saúde, bem-estar e envelhecimento: o estudo SABE no Município de São Paulo. Revista Brasileira de Epidemiologia, São Paulo, v. 8, n. 2, p. 127-141, jun. 2005. Disponível em: <http://www.scielo.br/scielo.php?script=sci_arttext \&pid=S1415-790X2005000200005>. Acesso em: 29 jan. 2007.

LIMA-COSTA, M. F.; BARRETO, S. M. Tipos de estudos epidemiológicos: conceitos básicos e aplicações na área do envelhecimento. Epidemiologia e Serviços de Saúde, Brasília, DF, v. 12, n. 4, p. 189201, 2003.

LIMA-COSTA, M. F. et al. Desigualdade social e saúde entre idosos brasileiros: um estudo baseado na pesquisa nacional por amostra de domicílios. Cadernos de Saúde Pública, Rio de Janeiro, v. 19, n. 3, p. 745-757, 2003 .

MARCON, S. S. et al. Características da doença crônica em famílias residentes na região norte de Maringá-PR: uma primeira aproximação. Acta Scientiarun, Maringá, v. 26, n. 1, p. 83-93, 2004. MATHIAS, T. A. F.; JORGE, M. H. P. M.; LAURENTI, R. Doenças cardiovasculares na população idosa: análise do comportamento da mortalidade em município da região Sul do Brasil no período de 1979 a 1998. Arquivos Brasileiros de Cardiologia, São Paulo, v. 82, n. 6, p. 533-541, jun. 2004. 
ORGANIZAÇÃO DAS NAÇÕES UNIDAS. Assembléia mundial sobre envelhecimento: resolução 39/125.

Viena, 1982.

ORGANIZAÇÃO MUNDIAL DA SAÚDE. Cuidados inovadores para as condições crônicas:

componentes estruturais de ação, relatório mundial 2003. Brasília, DF, 2003.

ROACH, S. Introdução à enfermagem gerontológica. Rio de Janeiro: Guanabara Koogan, 2003.

RODRIGUES, R. A. P.; DIOGO, M. J. D.; BARROS, T. R. O envelhecimento do ser humano. In: RODRIGUES, R. A. P.; DIOGO, M. J. D. (Org.). Como cuidar dos idosos. Campinas: Papirus, 1996. p. 11-16.

SHOUERI, J. R.; RAMOS, L. R.; PAPALÉO NETO, M. Crescimento populacional: aspectos demográficos e sociais. In: CARVALHO FILHO, E. T.; PAPALÉO NETO, M. Geriatria: fundamentos, clínica e terapêutica. São Paulo: Atheneu, 2000. p.9-29.

SILVA, M. C. O processo de envelhecimento no Brasil: desafios e perspectivas. Textos sobre Envelhecimento, Rio de Janeiro, v. 8, n. 1, 2005. Disponível em: <http://www.unati.uerj.br>. Acesso em: 20 set. 2005 .
SMELTZER, C. S.; BARE, G. Tratado de enfermagem médico-cirúrgica. 8. ed. Rio de Janeiro: Guanabara Koogan, 1998.

SOUZA, M. F. A enfermagem reconstruindo sua prática: mais que uma conquista no PSF.

RevistaBrasileira de Enfermagem, Brasília, DF, v. 53, p. 25-30, 200o. Número especial.

STEVENSON, J. S.; GONÇALVES, L. H. T.; ALVAREZ, A. M. O cuidado e a especificidade da enfermagem geriátrica e gerontológica. Texto e Contexto Enfermagem, Florianópolis, v. 6, n. 2, p. 33-55, maio/ ago. 1997.

TREVIZAN, M. A. Enfermagem hospitalar: administração e burocracia. Brasília, DF: UnB, 1988.

VERAS, R. A era dos idosos: desafios

contemporâneos. In: SALDANHA, A. L.; CALDAS, C. P. (Org.). Saúde do idoso: a arte de cuidar. 2 ed. Rio de Janeiro: Interciência, 2004. p.3-10.

VERMELHO, L. L.; MONTEIRO, M. F. G. Transição demográfica e epidemiológica. In: MEDRONHO, R. A. et al. Epidemiologia. São Paulo: Atheneu, 2002. p.91103. 